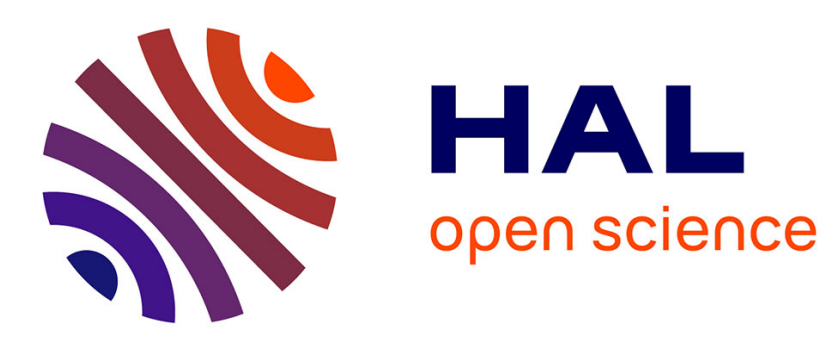

\title{
Diabatic pseudofragmentation and nonadiabatic excitation-energy transfer in meta-substituted dendrimer building blocks
}

\author{
Emmeline K.-L. Ho, Benjamin Lasorne
}

\section{- To cite this version:}

Emmeline K.-L. Ho, Benjamin Lasorne. Diabatic pseudofragmentation and nonadiabatic excitationenergy transfer in meta-substituted dendrimer building blocks. Computational and Theoretical Chemistry, 2019, 1156, pp.25-36. 10.1016/j.comptc.2019.03.013 . hal-02160459

\author{
HAL Id: hal-02160459 \\ https://hal.science/hal-02160459
}

Submitted on 21 Dec 2020

HAL is a multi-disciplinary open access archive for the deposit and dissemination of scientific research documents, whether they are published or not. The documents may come from teaching and research institutions in France or abroad, or from public or private research centers.
L'archive ouverte pluridisciplinaire HAL, est destinée au dépôt et à la diffusion de documents scientifiques de niveau recherche, publiés ou non, émanant des établissements d'enseignement et de recherche français ou étrangers, des laboratoires publics ou privés. 


\title{
Diabatic pseudofragmentation and nonadiabatic excitation-energy transfer in meta-substituted dendrimer building blocks
}

\author{
Emmeline K.-L. Ho and Benjamin Lasorne* \\ Institut Charles Gerhardt \\ UMR 5253 CNRS - Université de Montpellier - ENSCM \\ 34095 Montpellier, France
}

March 11, 2019

\begin{abstract}
Excitation-energy transfer in multichromophoric assemblies is often pictured in terms of excitonic models whereby the underlying diabatic states correlate with the ground (acceptor) and excited (donnor) electronic states of well-defined separated fragments. Poly(phenylene ethynylene) dendrimers also exhibit ultrafast unidirectional excitation-energy transfer; however, the definition of the fragments is no longer straightforward, as adjacent chromophores share a common meta-substituted phenylene ring, which cannot be viewed as a spectator bridge from an electronic perspective. Here, we show how a pseudofragmentation scheme can be used to define the relevant diabatic representation, provided the interacting sites are based on orbital subsets rather than atomic clusters. This is illustrated with the smallest meta-substituted oligomer, for which we characterised a conical intersection responsible of nonadiabatic internal conversion between the first two excited electronic states, consistent with a diabatic picture based on para-conjugated pseudofragments sharing a ring.
\end{abstract}

Keywords: excitation-energy transfer (EET), diabatic representation, nonadiabatic internal conversion, conical intersection, poly(phenylene ethynylene) (PPE) dendrimers.

\footnotetext{
*benjamin.lasorne@umontpellier.fr
} 


\section{Introduction}

A crucial aspect for any study involving so-called grid-based quantum dynamics is the availability - hence, the actual production in a preliminary stage - of a potential-energy surface (PES), given as an analytic, high-dimensional function of the nuclear coordinates. In the context of nonadiabatic dynamics, beyond the Born-Oppenheimer approximation, the electronic Hamiltonian for the subset of coupled electronic states is not reduced to a single of its eigenvalues, but rather expressed as a matrix, most often in a diabatic representation, with potential energies on the diagonal part and potential couplings on the off-diagonal part.

As opposed to what occurs for the adiabatic representation, the values of the diabatic matrix elements on the relevant set of molecular geometries are not computed directly from quantum-chemistry calculations and first depend on a nontrivial adiabatic-to-diabatic transformation, called diabatisation, of the quantum-chemistry data as a prerequisite. A review on this topic is out of the scope of the present work. Let us mention perhaps one of the most standard procedures in this context, resorting to what is known as a "diabatisation by ansatz", namely, the vibronic-coupling Hamiltonian model developed in the 1980s by Köppel et al., which has been used since then with great success in numerous quantum-dynamics simulations $[1,2]$.

By definition, diabatic states preserve their character (electronic distribution; in particular bonding pattern) when the molecular geometry changes. In molecular systems baring a single chromophore, such states will often be labelled according to dominant electronic configurations (e.g., $n \pi^{*}$ or $\pi \pi^{*}$, as in pyrazine [3]) or to the change in the electronic distribution with respect to the ground state (e.g., charge transfer (CT) or locally excited (LE), as in aminobenzonitrile $[4])$.

Although seemingly more complex, systems made of several weakly interacting chromophores, such as supramolecular light-harvesting antennae, yield diabatic states that are somewhat more obvious to define, as they correspond to local excitations that involve only one specific site at a time. In this context, excitation-energy transfer (EET) [5] if often pictured in terms of local excitonic models whereby each elementary act occurs from a donor site in its excited state to an neighbouring acceptor site in its ground state. The underlying states are essentially diabatic and correlate with the ground and excited electronic states of well-defined separated fragments. This is for example the basis for the fragment excitation difference (FED) scheme aimed at addressing EET in this context $[6,7]$.

In other words, EET among diabatic states can be regarded as a nonadiabatic process. Accounting for the motion of the nuclei means that EET essentially boils down to internal conversion, likely to occur around conical intersections or weakly avoided crossings among locally excited (LE) states within the whole system.

In much the same way, poly(phenylene ethynylene) (PPE) dendrimers exhibit ultrafast unidirectional EET through LE states. These are tree-like, hyperbranched multichromophoric macromolecules, designed to mimic natural photo- 
synthesis artificially. The most famous one is called the nanostar and was first synthesised by J. S. Moore et al. in 1994 [8].

Dendrimers present a huge potential in the context of organic nano-optoelectronics $[9,10]$. They are made of building blocks of increasing length from the periphery to the core, which yields an excitation-energy gradient responsible of unidirectional energy transduction along several, converging branched pathways, inducing fluorescence-efficiency enhancement of the fluorophore at the core $[11,12,13,14]$.

Much effort has been made over the last thirty years to unravel the mechanisms governing the behaviour of PPEs on the atomistic scale. First insights into the electronic structure of PPEs were provided by steady-state spectroscopy experiments $[12,15]$. These established that the absorption spectrum of the nanostar is almost additive and dominated by individual contributions of $\pi \pi^{*}$ LE states on linear para-conjugated building blocks. This was later confirmed by other experimental and theoretical studies on various types of PPE dendrimer subunits; see e.g. Refs. [16, 17, 18, 19, 20, 21, 22] and references therein.

However, the definition of the coupled fragments in PPE dendrimers is no longer straightforward, since adjacent chromophores share a common metasubstituted phenylene, which cannot be viewed as a spectator bridge [23]. Here, we show that a pseudofragmentation scheme is able to generate the relevant diabatic representation, provided the definition of the sites is based on orbital subsets rather than atomic clusters. This is illustrated with the smallest meta-substituted oligomer, meta-diphenylethynylphenylene, for which we characterised a conical intersection likely to be responsible of ultrafast and efficient internal conversion between the first two excited electronic states.

The energy landscapes of the first two excited electronic states are first described, including minima, transition states, and a minimum-energy conical intersection. A discussion of the relevant molecular orbitals in then provided, setting the foundations for a description of the adiabatic states in terms of diabatic states corresponding to pseudofragments sharing a common ring. 


\section{Computational Details}

All calculations were performed with the Gaussian16 package (revision A03) [24] using DFT (ground states) and TD-DFT (excited states) at the CAM-B3LYP /6$31+\mathrm{G}^{*}$ level of theory, the validity of which having been already assessed in a previous work [25] on para-conjugated PPE oligomers with two, three, and four rings, yielding vibronic absorption spectra in excellent agreement with experiments (DFT: density-functional theory; TD-DFT: time-dependent DFT, i.e, first-order linear-response DFT). LE states (the focus of the present work) in meta-substituted oligomers are thus expected to be described adequately at this level of theory. However, states with large CT contributions may be overstabilised, which is well known, and their energies should be considered with caution, as for example pointed out in this context in Ref. [26].

Minima and transition states were obtained and characterised with analytic first- and second-derivative calculations in the ground and excited electronic states. The minimum-energy conical intersection within the $\mathcal{C}_{2 \mathrm{v}}$ subspace was obtained upon minimising the gradient average, projected out of the gradient difference. Both are totally symmetric, while the derivative coupling here is not; we did not look for broken-symmetry conical intersections, as the energy landscape in the vicinity of the $\mathcal{C}_{2 \mathrm{v}}$ minimum-energy conical intersection seems to indicate that this is the lowest-energy crossing point. The branching-space vectors of the minimum-energy conical intersection (directions of first-order degeneracy lifting) are the (halved) gradient difference (obtained directly from the two gradients) and the derivative coupling at this point. The latter was generated from a two-Hessian-based, "wavefunction free", procedure, detailed in Ref. [27]. Molecular orbitals displayed in the following are canonical Kohn-Sham DFT orbitals (0.02 a.u. isovalue surface plots). The Cartesian coordinates of all critical points discussed below are provided in Supplementary Information.

For the $\mathcal{C}_{2 \mathrm{v}}$ point group of symmetry, we used Mulliken's convention, so that $z\left(A_{1}\right)$ is the $C_{2}$ rotation axis, $y\left(B_{2}\right)$ lies within the molecular plane (leftright direction), and $x\left(B_{1}\right)$ is orthogonal to it (up-down direction). Only in-

plane deformations were considered, and the $\mathcal{C}_{\mathrm{s}}$ point group of lower symmetry mentioned below corresponds to mirror symmetry with respect to the molecular plane $\left(A_{1}\right.$ and $B_{2}$ both become $\left.A^{\prime}\right)$. 


\section{Results and Discussion}

\subsection{Multistate Energy Landscape}

Here, we characterise the energy landscape corresponding to the first and second excited singlet electronic states, $S_{1}$ and $S_{2}$, of the meta-diphenylethynylphenylene species, denoted m22 from now on. In particular, we show how the symmetry breaking at the $S_{1}$ equilibrium geometry, and subsequent electronic localisation on either one of the two equivalent sides, is consistent with the presence of a conical intersection between $S_{1}$ and $S_{2}$.

The m22 species belongs to the $\mathcal{C}_{2 \mathrm{v}}$ point group of symmetry at the $S_{0}$ equilibrium geometry, $\min _{S_{0}}$; see Figure 1 . The values of the internal coordinates are almost identical to those of the 2-ring linear species (diphenylethynylene, also known as diphenylacetylene or tolane, further on denoted p2) in its ground electronic state, as illustrated for the bond lengths in Table 1, exhibiting a typical alternation of single-triple-single $\mathrm{CC}$ bonds between rings. Note that the small differences for the central phenylene ring indicate that it behaves as if it had been rotated through $\pm 60^{\circ}$ to account for symmetrical meta-substitution.

At this geometry, also called Franck-Condon (FC) point, the $S_{1}$ and $S_{2}$ states are almost degenerate: the corresponding vertical transition energies are 4.43 and $4.47 \mathrm{eV}$, respectively. The $S_{0}$ state is $1^{1} A_{1}$, the $S_{1}$ state $1^{1} B_{2}$ (bright; oscillator strength: 1.707 ), and the $S_{2}$ state $2^{1} A_{1}$ (bright; oscillator strength: 0.367 ). Further details on the electronic structure will be given below, in Sec. 3.3. $S_{1}$ and $S_{2}$ can be identified to two diabatic states, determined by symmetry, thus noninteracting over the whole $\mathcal{C}_{2 \mathrm{v}}$ subspace. We will show later on that these delocalised diabatic states can be recast as the normalised sum and difference of two localised diabatic states. The latter two both involve local excitations of either the "left" or "right" p2 subunits contained within m22. In addition, we will rationalise how these can be understood in terms of a pseudofragmentation scheme, whereby the pseudofragments share the meta-substituted central ring.

The two excited states cross within the $\mathcal{C}_{2 \mathrm{v}}$ subspace. The minimum-energy conical intersection within this subspace, denoted $\operatorname{CoIn}_{S_{2} / S_{1}}$, occurs at $4.29 \mathrm{eV}$ above the $S_{0}$ minimum (see Figure 6). It is lower in energy than the FC point in $S_{2}$, and thus potentially accessible (see Table 1). Two transition states in the $S_{1}$ PES lye on either side of the conical intersection, each behaving as an apparent minimum within the $\mathcal{C}_{2 \mathrm{v}}$ subspace. One corresponds to $1^{1} B_{2}$ at $4.25 \mathrm{eV}$ (same side as the FC point), the other to $2^{1} A_{1}$ at $4.29 \mathrm{eV}$ (beyond the crossing). They will further be denoted $\mathrm{TS}_{B_{2}}$ and $\mathrm{TS}_{A_{1}}$, respectively (see Table 1). The $\mathrm{TS}_{A_{1}}$ point is almost identical to the $\mathrm{CoIn}_{S_{2} / S_{1}}$ point. More precisely, $\mathrm{CoIn}_{S_{2} / S_{1}}$ is essentially the same as the crossing point occurring along the interpolation path in internal coordinates between $\mathrm{TS}_{B_{2}}$ and $\mathrm{TS}_{A_{1}}$, near $\mathrm{TS}_{A_{1}}$ (see below) where the gradient average, projected out of the gradient difference, is already small before further optimisation.

Owing to the respective PES topographies, the $\mathrm{CoIn}_{S_{2} / S_{1}}$ point also occur to play the role of the actual minimum (conical, but not stationary, due to the two-dimensional cusp) of the $S_{2}$ PES. The gradient difference calculated 
at the conical intersection essentially corresponds to the direction connecting $\mathrm{TS}_{A_{1}}$ on one side to $\mathrm{TS}_{B_{2}}$ on the other side. As will be shown soon, the derivative coupling is essentially the direction leading to the pair of equivalent lower-symmetry $S_{1}$ minima.

The main difference between the geometry of $\mathrm{TS}_{B_{2}}$ and $\mathrm{TS}_{A_{1}}$ is localised on the central ring, in particular the length of its quinoidal bonds, which is shorter for $\mathrm{TS}_{B_{2}}$ (see Table 1). They can be represented in terms of Lewis structures as a biradical quinoidal ring (Dewar-benzene bonding) for $\mathrm{TS}_{B_{2}}$, and a combination of two single-bond-linked allyl radicals in $\mathrm{TS}_{A_{1}}$; see Figure 2. This agrees with the topologies of the relevant near-frontier orbitals (see Sec. $3.2)$.

The transition vector (unstable mode) of each transition state is of $B_{2}$ symmetry (in-plane, left-right symmetry breaking). Both are displayed in Figure 3. They directly connect the two mirror-image $S_{1}$ minima of $\mathcal{C}_{\mathrm{s}}$ symmetry on both sides of the $S_{1}$ unstable ridge, further denoted $\min _{S_{1}}$ and $\min _{S_{1}}^{\prime}$ (see Figure 1 and Table 1). At each of these two equivalent minima, the system shows an alternation of single-triple-single CC bonds on one side (similar to a groundstate p2 moeity) and a cumulenic (double-double-double) bonding scheme on the other one, typical of an excited-state p2 moiety (see Figure 1 and Table 1 ). In other words, such geometries reflect two equivalent local excitations, on either one or the other p2 pseudofragment sharing the same meta-substituted central ring.

Energy pathways, obtained as linear interpolations in internal coordinates, between the aforementioned points are shown in Figure 5. They are smooth, nearly harmonic, energy curves, which confirms that the relevant "anchor points" of the two-state energy landscape have all extensively been identified within the operational energy window accessible from the FC point. By this, we mean that the representative topographies, governed by local-curvature variations, of the $S_{2}$ and $S_{1}$ PESs, are fully determined by the presence of both $\mathrm{TS}_{B_{2}}$ and $\mathrm{TS}_{A_{1}}$ on the same higher-symmetry ridge within the $\mathcal{C}_{2 \text { v }}$ subspace. Each occurs on either side of the minimum-energy $\mathrm{CoIn}_{S_{2} / S_{1}}$ point, and both are connected directly - downhill to either $\min _{S_{1}}$ or $\min _{S_{1}}^{\prime}$, which are mirror-image minima $\left(\mathcal{C}_{\mathrm{s}}\right.$ symmetry). This ideal picture was confirmed quantitatively with steepestdescent paths originated from the FC point. First, minimising the $S_{2}$ energy from the FC point directly leads to the $\mathrm{CoIn}_{S_{2} / S_{1}}$ point. Second, the intrinsicreaction coordinate started along the $S_{2}$ gradient also goes directly to the $\mathrm{TS}_{A_{1}}$ point via the $\operatorname{CoIn}_{S_{2} / S_{1}}$ crossing. It further continues on $S_{1}$ to one of the two broken-symmetry minima. A more detailed analysis of the deactivation mechanism awaits quantum-dynamics simulations, which will be addressed in a future work.

When approaching either one of the two $S_{1}$ minima, one can notice a weakly avoided crossing between $S_{2}$ and $S_{3}$ (around $4.6 \mathrm{eV}$ ), which is expected to be of little consequence on the photodynamics, once the system has escaped the original region of near-crossing between $S_{2}$ and $S_{1}$ (especially owing to the fact that it lies higher in energy than the FC point on $S_{2}$, at about $4.5 \mathrm{eV}$ ).

In principle, initial excitation will populate preferentially $S_{1}$, given its larger 
oscillator strength. However, we are specifically interested here in the evolution of the part of the system starting from $S_{2}$, as a prototype of nonadiabatic energy transfer in dendrimers. A schematic sketch of the plausible deactivation pathway from $S_{2}$ to $S_{1}$ is displayed with arrows in Figure 5. After excitation to the $S_{2}$ state at the FC point, the system will descend along a very narrow trench in the $S_{2}$ state towards the region around the minimum-energy conical intersection, which is expected to act as an efficient "kitchen sink" to the $S_{1}$ state. As the energy difference is low all along this pathway, quantum or semiclassical dynamics should predict large and early population transfer. In fact, this case is quite exceptional, as the energy difference is significantly small $-<0.1 \mathrm{eV}$ - already from the original FC point, and a semiclassical description such as surface hopping may be at risk regarding the relevance of a supposedly high hopping repetition rate to be damped by a fewest-switch scheme.

Once in the region near the conical intersection at the bottom of the $S_{2}$ PES, the system can branch to two sides when keeping $\mathcal{C}_{2 \mathrm{v}}$ symmetry: turning slightly aside to the $\mathrm{TS}_{B_{2}}$ region on the same side of the crossing, whereby the energy difference will increase as much as possible, thus separating efficienlty the $S_{1}$ wavepacket component from the original $S_{2}$ one, or keeping to the $\mathrm{TS}_{A_{1}}$ region, on the other side but not far from the crossing region, which is likely to act as a turning point and send the system back again to the original side for further crossings. In any case, it must be understood that the $S_{1}$ PES in this region essentially corresponds to a very narrow unstable ridge. The adiabatic wavepacket component on $S_{2}$ should thus spread strongly and early on because it lives in a very narrow trench; correlatively, the wavepacket component transferred from $S_{2}$ to $S_{1}$ will land on a very steep ridge, and is expected to also keep spreading very fast on $S_{1}$, so as to reach broken-symmetry regions typical of the $S_{1}$ minimum in a short time. In other words, the nonadiabatic coupling between $S_{2}$ and $S_{1}$ is expected to be exceptionnally strong and efficient. The present case is typically far from a Born-Oppenheimer situation, which makes the adiabatic Born-Oppenheimer representation almost inadequate (in other words, the two electronic states are too close in energy).

The surroudings of the conical intersection within the branching space are displayed in Figure $6\left(S_{2}\right.$ and $S_{1}$ energies along the plane spanned by the gradient difference and derivative coupling). Geometric relaxation is obviously not fully achieved for the $\mathrm{TS}_{B_{2}}$ and $\mathrm{TS}_{A_{1}}$ points, or for the $\min _{S_{1}}$ and $\min _{S_{1}}^{\prime}$ ones, within this subspace, but the energy landscape is representative of the $S_{2}$ and $S_{1}$ PES topographies: the conical intersection is the actual $S_{2}$ minimum; there are two $S_{1}$ transition states along the gradient difference (one on each side of the conical intersection) connecting two equivalent $S_{1}$ minima on both sides, along the derivative coupling. 

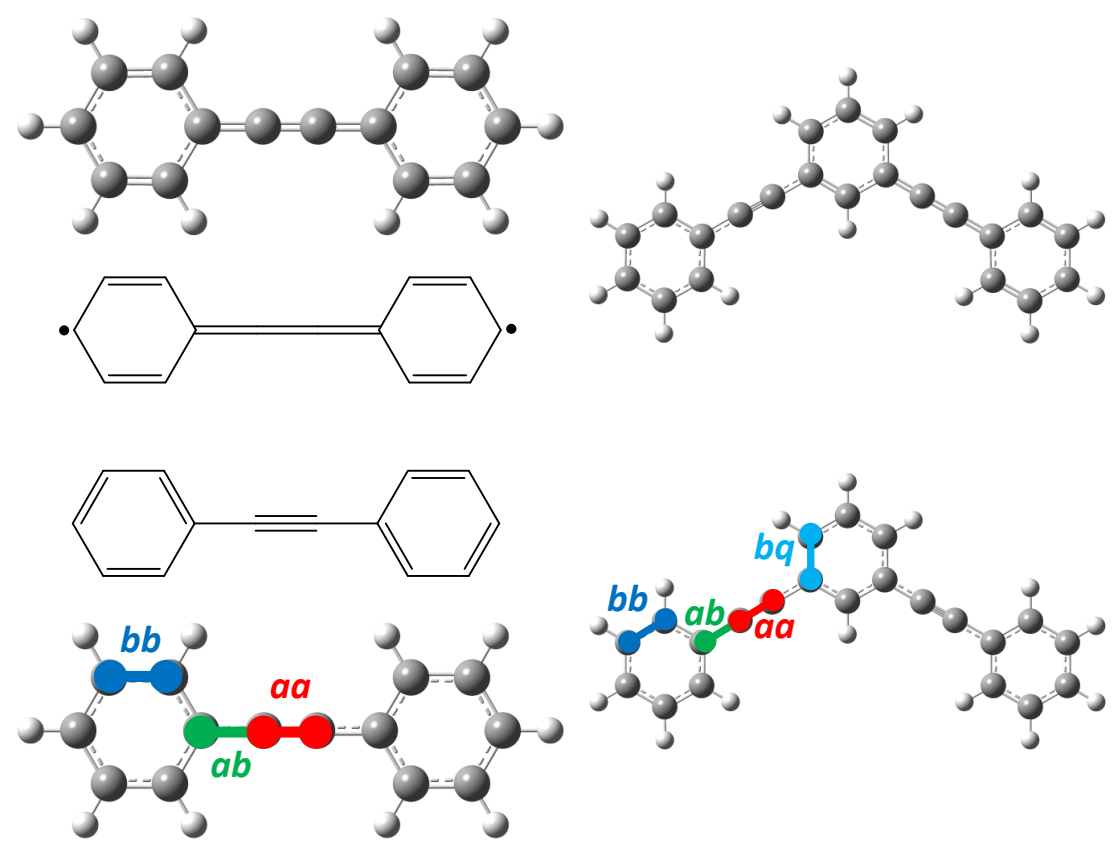

Figure 1: $S_{0}$ (bottom) and $S_{1}$ (top) equilibrium geometries of m22 (right) and p2 (left). Representative bonds are labelled according to bond lengths given in Table 1 . The typical bonding schemes $\left(S_{0}\right.$ alternated, bottom; $S_{1}$ cumulenic, top) of p2 are also indicated for reference.

\begin{tabular}{lcccccccc} 
& $a a$ & $a b$ & $b b$ & $b q$ & $E_{0}$ & $E_{1}$ & $E_{2}$ & $E_{3}$ \\
\hline $\mathrm{p} 2 S_{0} \min$ & 1.210 & 1.431 & 1.389 & & 0 & 4.48 & & \\
\hline $\mathrm{p} 2 S_{1} \min$ & 1.255 & 1.374 & 1.377 & & 0.32 & 4.14 & & \\
\hline $\mathrm{m} 2 \mathrm{~min}_{S_{0}}$ & 1.210 & 1.431 & 1.389 & 1.402 & 0 & 4.43 & 4.47 & 4.61 \\
\hline $\mathrm{m} 2 \min _{S_{1}} / \min _{S_{1}}^{\prime}$ & $1.210 / 1.254$ & $1.429 / 1.374$ & $1.389 / 1.377$ & $1.415 / 1.438$ & 0.32 & 4.12 & 4.66 & 4.74 \\
\hline $\mathrm{m} 2 \mathrm{TS}_{B_{2}}$ & 1.233 & 1.396 & 1.381 & 1.419 & 0.18 & 4.25 & 4.33 & 4.52 \\
\hline $\mathrm{m} 2 \mathrm{TS}_{A_{1}}$ & 1.229 & 1.405 & 1.384 & 1.447 & 0.19 & 4.29 & 4.30 & 4.53 \\
\hline $\mathrm{m} 2 \mathrm{CoIn}_{S_{2} / S_{1}}$ & 1.230 & 1.404 & 1.384 & 1.444 & 0.19 & $4.29^{*}$ & $4.29^{*}$ & 4.52 \\
\hline
\end{tabular}

Table 1: Selection of representative bond lengths (in $\AA$ ) and corresponding energies (in $\mathrm{eV}$ ) of the first four electronic states of $\mathrm{m} 22$ at the five critical points discussed in the main text. Similar quantities are provided for the $S_{0}$ and $S_{1}$ minima of 2 for comparison. Absolute energies at both $S_{0}$ minima: -846.19837 hartree (m22); -539.15684 hartree (p2). * The energy difference at the $S_{2} / S_{1}$ conical intersection is $5.10^{-4} \mathrm{eV}$. 

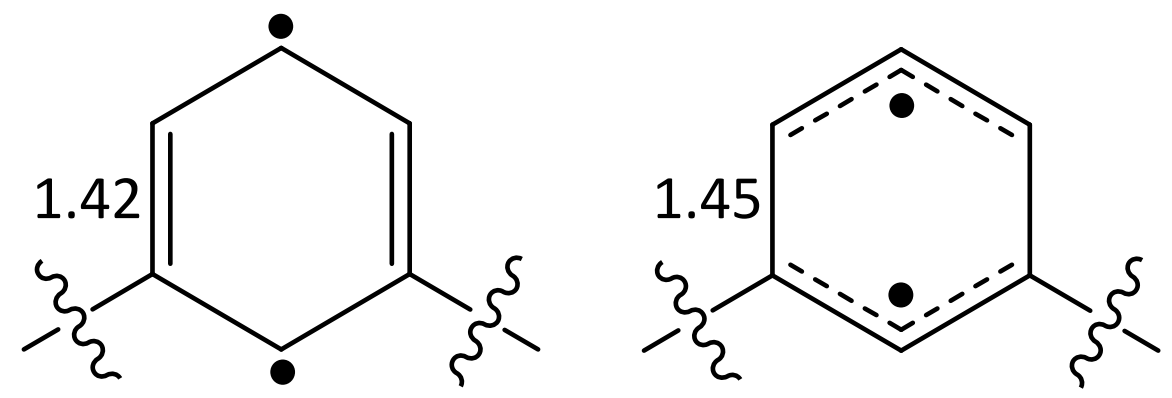

Figure 2: Lewis representations of the bonding schemes on the meta-substituted phenylenes of $\mathrm{TS}_{B_{2}}$ (left) and $\mathrm{TS}_{A_{1}}$ (right). Quinoidal bond lengths are given in $\AA$. 

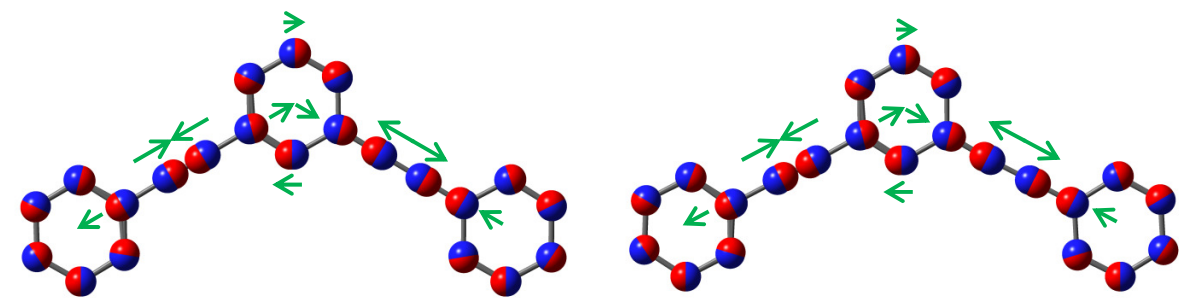

Figure 3: Transition modes of $\mathrm{TS}_{B_{2}}$ (left) and $\mathrm{TS}_{A_{1}}$ (right). The original positions of the $\mathrm{C}$-atoms are in blue, the displaced ones in red (H-atoms are not shown, as they barely move). Green arrows are schematic representations of the dominant motions. The corresponding imaginary frequencies are $\bar{\nu}\left(\mathrm{TS}_{B_{2}}\right)=\mathrm{i} 4891 \mathrm{~cm}^{-1}$ and $\bar{\nu}\left(\mathrm{TS}_{A_{1}}\right)=\mathrm{i} 15119 \mathrm{~cm}^{-1}$ (the latter is unusually high because of the presence of a conical intersection in the vicinity of $\operatorname{TS}_{A_{1}}$ ). 

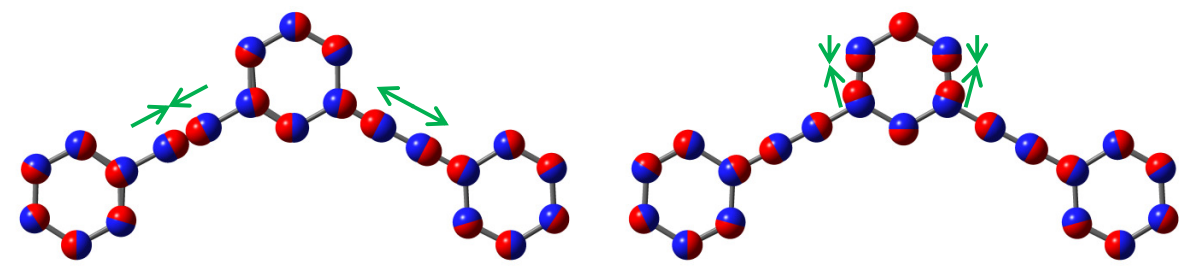

Figure 4: Derivative coupling (left) and gradient difference (right) calculated at $\mathrm{CoIn}_{S_{2} / S_{1}}$. The original positions of the C-atoms are in blue, the displaced ones in red (H-atoms are not shown, as they barely move). Green arrows are schematic representations of the dominant motions. 

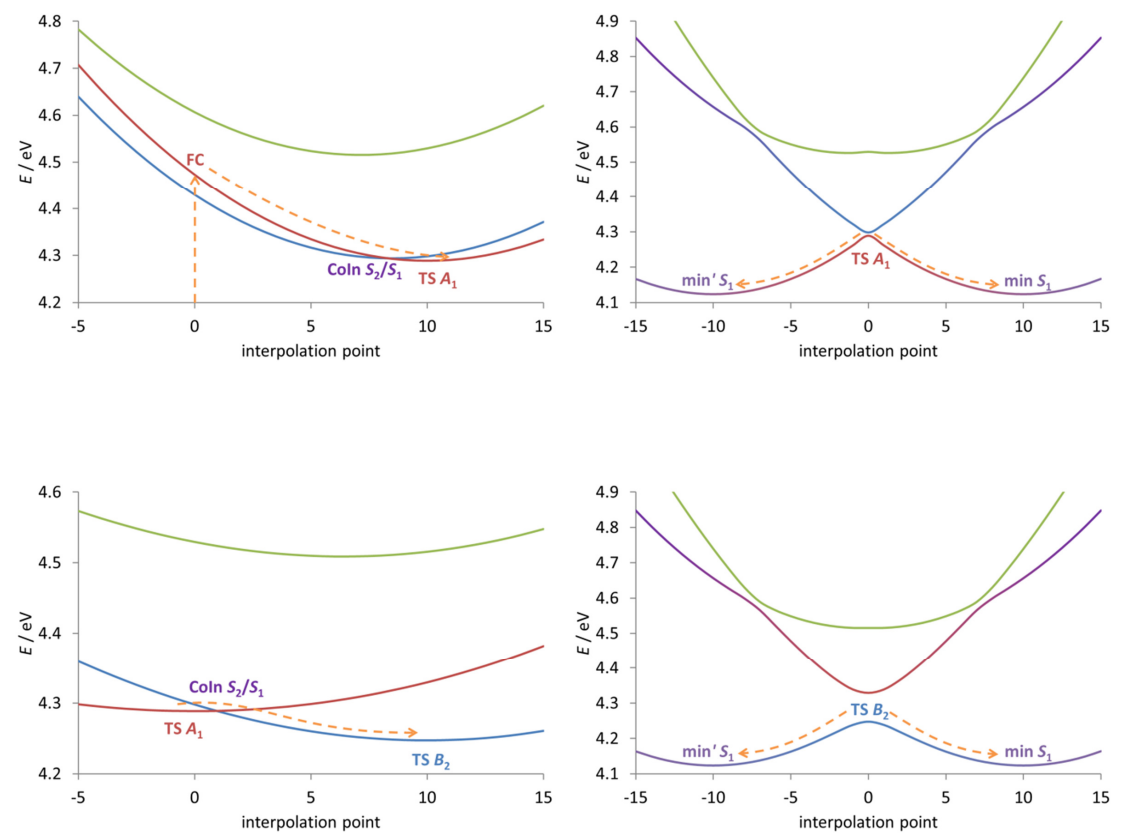

Figure 5: Linear interpolations (from 0 to 10), augmented with extrapolations (beyond 0 to 10), in internal coordinates between the most relevant critical points (at either 0 or 10 ). Blue: $2^{1} A_{1}\left(\mathcal{C}_{2 \mathrm{v}}\right), S_{2}$ or $S_{1}$; red: $1^{1} B_{2}\left(\mathcal{C}_{2 \mathrm{v}}\right), S_{1}$ or $S_{2}$; purple: ${ }^{1} A^{\prime}\left(\mathcal{C}_{\mathrm{s}}\right), S_{1}$ or $S_{2}$; green: $S_{3}$. Orange arrows: schematic deactivation pathway. 


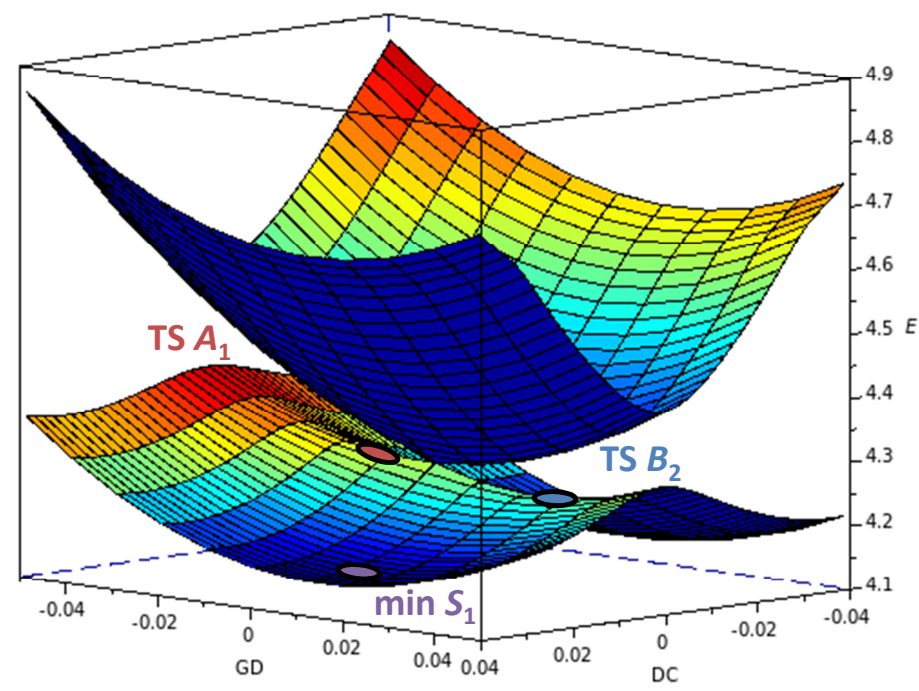

Figure 6: Conical intersection between the $S_{2}$ and $S_{1}$ PESs within the branching space (GD: (halved) gradient difference; DC: derivative coupling). Cumulative displacements over the whole set of nuclei along both gradient-type directions are given in $\AA$; energies are given in eV. The projected positions of $\mathrm{TS}_{B_{2}}$ (blue), $\mathrm{TS}_{A_{1}}$ (red), and $\min _{S_{1}}$ (purple) are marked with oval dots. 


\subsection{Pseudofragment Orbitals}

The excited electronic states of $\mathrm{m} 22$ can be rationalised via a four-electronin-four-orbital model. Below, we show how the four near-frontier molecular orbitals (HOMO-1, HOMO, LUMO, LUMO+1) of m22 naturally arise from two equivalent pairs of frontier orbitals (HOMO, LUMO) corresponding to the two embedded p2 pseudofragments on the "right" or "left" within m22, sharing a common ring (HOMO: highest-occupied molecular orbital; LUMO: lowestunoccupied molecular orbital).

As already pointed out in Ref. [23], the four near-frontier delocalised orbitals of $\mathrm{m} 22$ at the $C_{2 \mathrm{v}} S_{0}$ minimum can be obtained from a rotation of two equivalent pairs (HOMO, LUMO) of localised orbitals, on each p2 chromophore. Ignoring orthogonalisation tails due to nonzero overlaps, such delocalised orbitals simply are the normalised sum and diffferences of localised ones ( $45^{\circ}$-rotation). The HOMO and LUMO of m22 are of $\pi$-type and of symmetry $a_{2}$, while the HOMO-1 and LUMO +1 also are of $\pi$-type, and of symmetry $b_{1}$; see Figure 7 . The orbitals of $a_{2}$ symmetry are "minus" combinations of $\mathrm{p} 2$ frontier orbitals, while $b_{1}$ symmetry corresponds to "plus" combinations. The corresponding orbital diagram is depicted in Figure 7. Let us recall here that Mulliken's axis convention was used here for $\mathcal{C}_{2 \mathrm{v}}$ : $z\left(A_{1}\right)$ is the $C_{2}$ rotation axis, $y\left(B_{2}\right)$ lies within the molecular plane, and $x\left(B_{1}\right)$ is orthogonal to it.

The frontier orbitals of p2 (see Figure 8) essentially involve first-neighbour interactions between benzene and acetylene frontier $\pi$ - and $\pi^{*}$-orbitals: allantibonding (most destabilised) benzene-acetylene frontier-orbital interactions within the p2 HOMO, and all-bonding (most-stabilised) benzene-acetylene frontierorbital interactions within the p2 LUMO. The frontier $\pi$ - and $\pi^{*}$-orbitals of a single benzene form two pairs of degenerate orbitals. Only one per pair can be combined with the nearest acetylene $\pi$ - or $\pi^{*}$-orbital in p2, for symmetry reasons.

The pairs of delocalised orbitals of $\mathrm{m} 22$ are nearly but not degenerate, due to weak interactions between the orbitals localised on p2 pseudofragments (see Figure 7). The interaction magnitude can be related to the overlap between $\mathrm{p} 2$ orbitals. For a bare benzene, the overlap between two degenerate, meta-oriented orbitals $\left( \pm 60^{\circ}\right.$-rotated), either HOMO or LUMO, is equal to $-\frac{1}{2}$ (see Figure 9 ). The overlap between p2 orbitals is obviously closer to zero, as they expand over a larger space, thus explaining that HOMO and HOMO-1 (or LUMO and $\mathrm{LUMO}+1$ ) are nearly degenerate in $\mathrm{m} 22$.

This essentially reminds of a fragmentation scheme (the limiting case being a diatom for which the molecular orbitals are linear combinations of overlapping atomic orbitals). However, there is a significant difference here: the localised orbitals involve subsets of atoms (fragments) that have in common the central phenylene ring. This is the very reason why we call the two p2 moeities "pseudofragments". The contributions on the central phenylene essentially correspond to the normalised sum and difference of two degenerate, but nonorthogonal, benzene orbitals oriented along either the right or the left phenylethynylene substituent; see Figure 9. In other words, the fact that the central phenylene 
ring can bring contributions from two degenerate HOMOs and two degenerate LUMOs within meta-substitution is the key explanation: a meta-substituted phenylene somewhat behaves as a twofold site (within the framework of a firstneighbour, tight-binding picture). In contrast, a para-substituted phenylene behaves as a standard site, involving only one local HOMO for the global HOMO or one local LUMO for the global LUMO, for symmetry reasons, the other staying noninteracting, hence nonbonding.

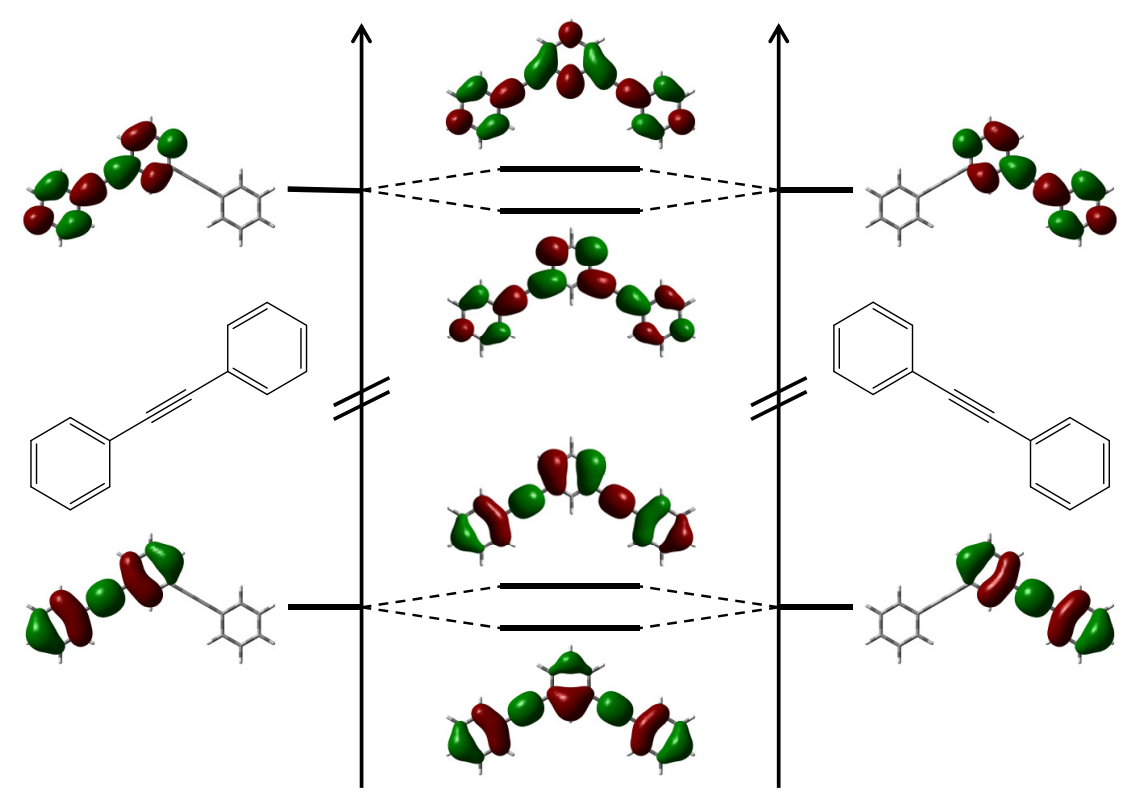

Figure 7: Orbital diagram describing the interactions between the frontier orbitals of the pseudo-fragments p2 to form the orbitals of m22 at the symmetrical $S_{0}$ minimum. The localised orbitals $\left(\ell, \ell^{*}, r, r^{*}\right)$ on the left- or right-hand side were obtained via a $45^{\circ}$-rotation from the delocalised Kohn-Sham molecular orbitals $\left(b_{1}, a_{2}, a_{2}^{*}\right.$, and $\left.b_{1}^{*}\right)$ displayed in the central part.

Let us now consider one of the two equivalent $S_{1}$ minima. The left-right equivalence is lost between the two p2 pseudofragments: one of them is in its ground-state geometry (alternated), while the other one is in its excited-state geometry (cumulenic). The two pairs of near-frontier orbitals become polarised, i.e., partly localised, as they no longer result from interactions between orbitals of the same energy (see Figure 10).

The energy order of the orbitals within a pair is consistent with their respective localisations on a given side. On Figure 11, the excited pseudofragment is on the right-hand side. The geometry of the pseudofragment on the left corresponds to the ground-state equilibrium geometry of p2 (single-triplesingle), while that on the right to the excited-state equilibrium geometry of $\mathrm{p} 2$ 


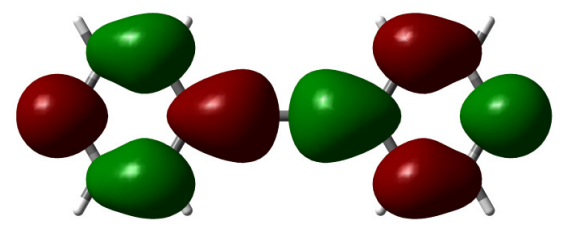

$S_{0} \min$ LUMO

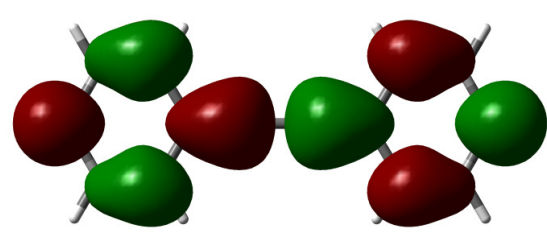

$S_{1} \min$ LUMO
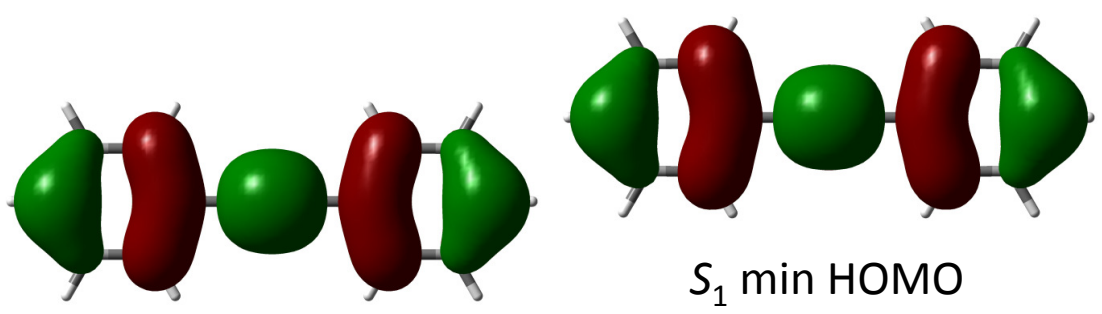

$S_{1} \min \mathrm{HOMO}$

$S_{0}$ min HOMO

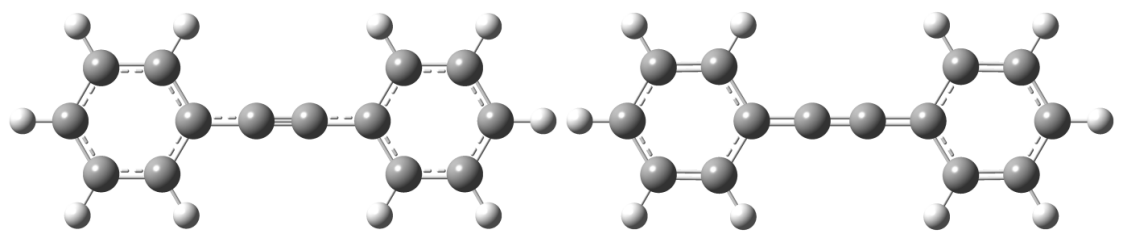

$S_{0}$ min geometry

$S_{1}$ min geometry
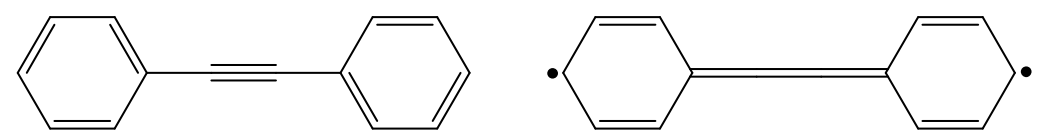

$S_{0}$ min bonding (alternated)

$S_{1}$ min bonding (cumulenic)

Figure 8: Geometries, bonding patterns, and frontier orbitals of an isolated p2 at its $S_{0}$ and $S_{1}$ minima. 

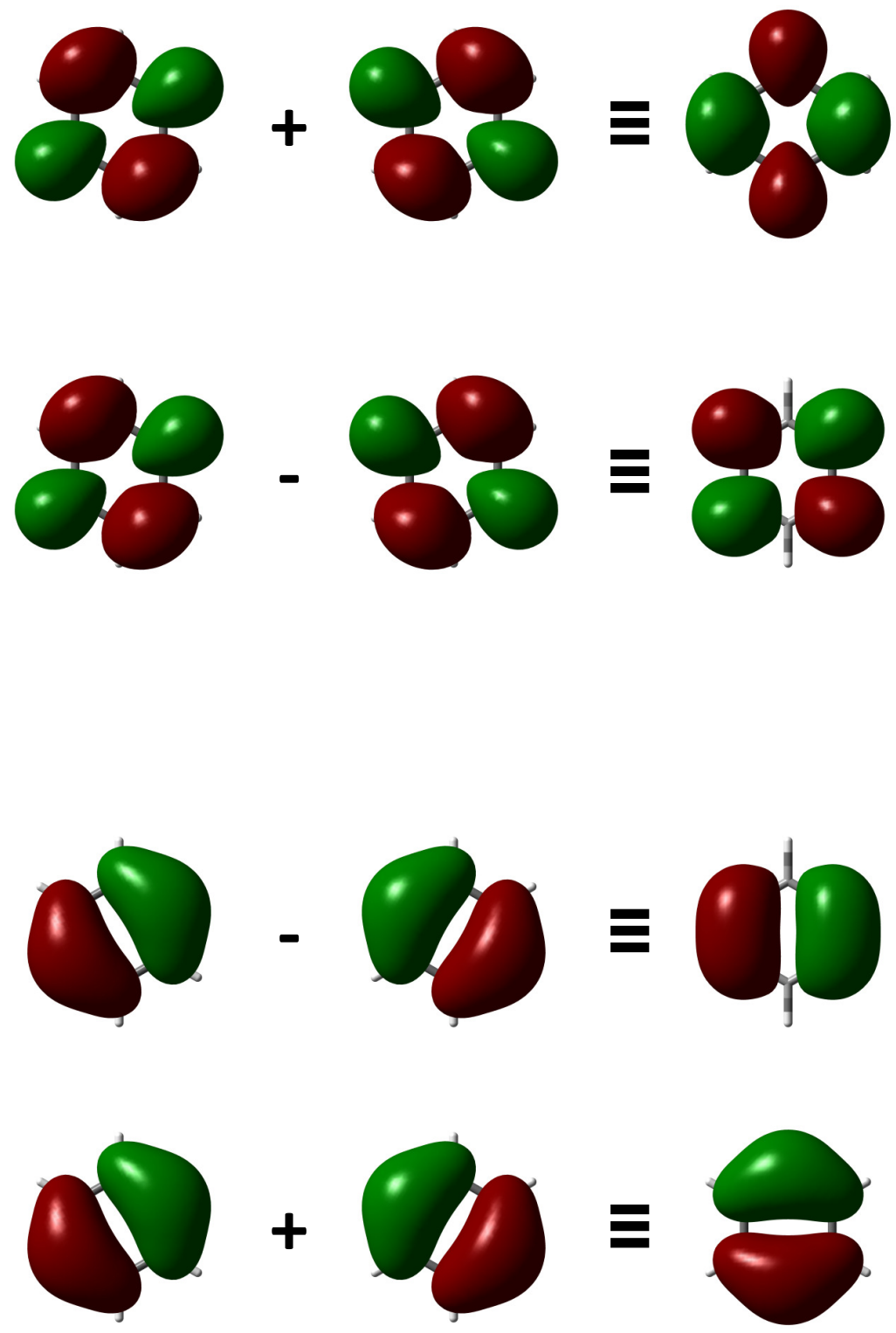

Figure 9: Decomposition of the two degenerate pairs of frontier $\pi$ - and $\pi^{*}$ orbitals of benzene in terms of combinations of nonorthogonal meta-oriented orbitals $\left( \pm 60^{\circ}\right.$-rotated). Normalisation factors are $N_{ \pm}=\frac{1}{\sqrt{1 \pm S}}$ (according to the sign of the combination); the overlap is equal to $S=-1 / 2$ in both cases, such that $N_{+}=1$ and $N_{-}=\frac{1}{\sqrt{3}}$. 
(double-double-double). For the occupied orbitals, the first-neighbour interactions between atomic orbitals along the $\mathrm{CCCC}$ chain between two rings are: antibonding-bonding-antibonding. An alternated bonding pattern is thus stabilised by single-triple-single bond lengths: HOMO-1 (stabilised) is localised on the unexcited pseudofragment (alternated), while HOMO (destabilised) is localised on the excited one (cumulenic). In contrast, the interactions for the unoccupied orbitals are: bonding-antibonding-bonding. A cumulenic bonding pattern is thus stabilised by double-double-double bond lengths: LUMO (stabilised) is localised on the excited pseudofragment (cumulenic), while LUMO+1 (destabilised) is localised on the unexcited one (alternated). As a result, the $S_{1}$ state at the $S_{1}$ minimum essentially corresponds to a single HOMO $\rightarrow$ LUMO excitation, and the electronic redistribution is consistent with the geometrical changes.

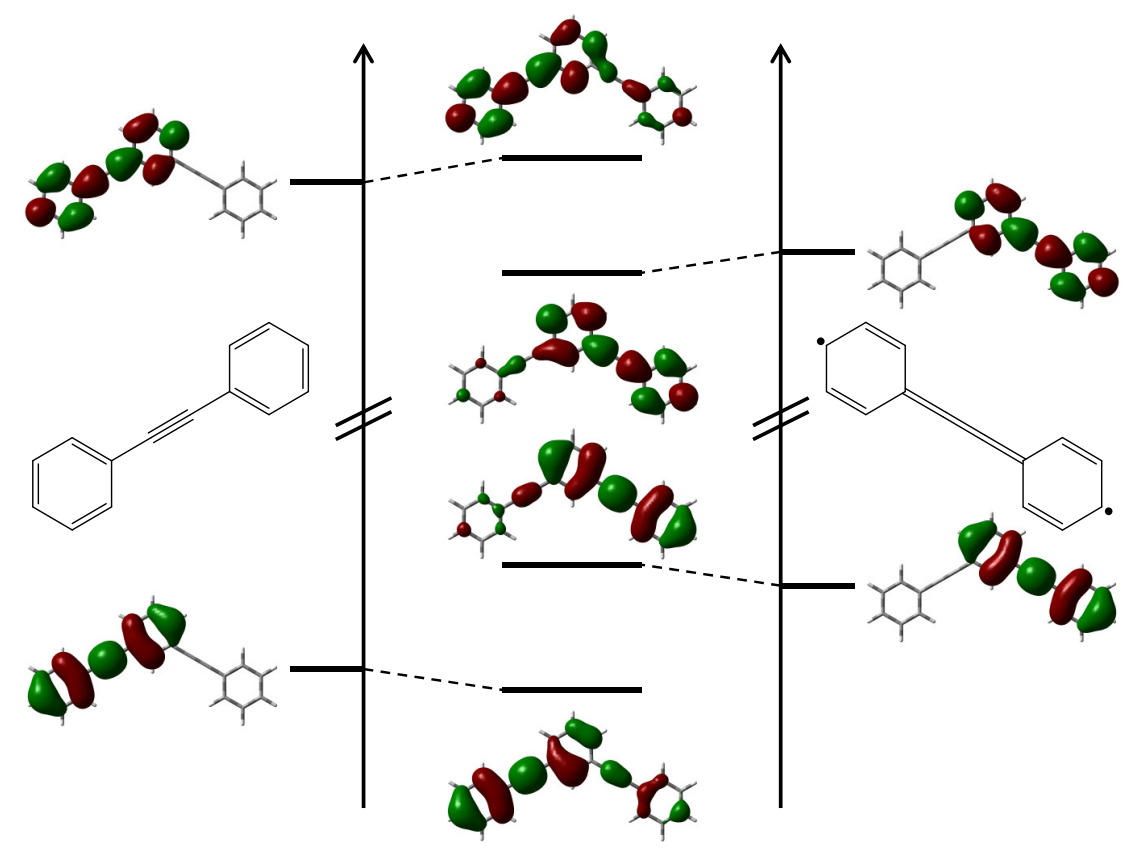

Figure 10: Orbital diagram describing the interactions between the frontier orbitals of the pseudo-fragments p2 to form the orbitals of $\mathrm{m} 22$ at the nonsymmetrical $S_{1}$ minimum (alternated on the left, cumulenic on the right). The Kohn-Sham molecular orbitals $\left(\sim \ell, \sim r, \sim r^{*}, \sim \ell^{*}\right)$ displayed on the central part are approximately localised. The localised orbitals $\left(\ell, \ell^{*}, r, r^{*}\right)$ on the leftor right-hand side are those from Figure 7. 

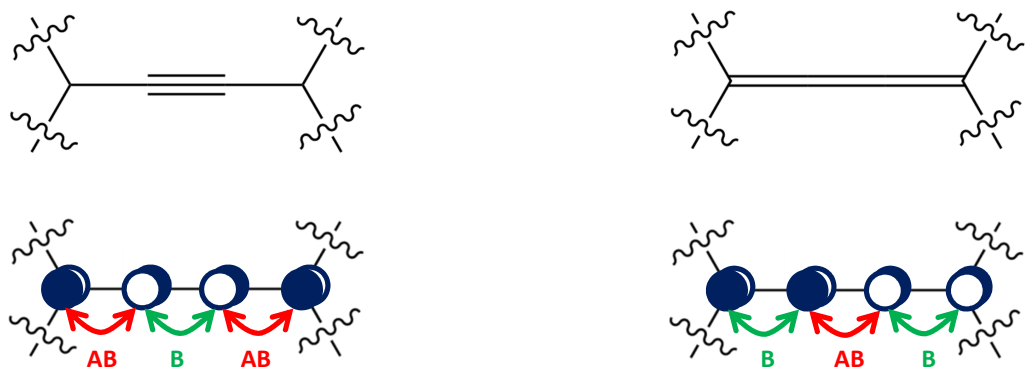

Figure 11: Bonding schemes along a CCCC chain and interpretation in terms of first-neighbour interactions between $p$ orbitals; alternated bonding scheme (left), cumulenic bonding scheme (right). B stands for bonding interactions and $\mathrm{AB}$ for antibonding interactions. 


\subsection{Diabatic States}

We now analyse the nature of the excited states and define delocalised and localised diabatic states from the adiabatic ones, according to the pseudofragmention scheme presented above.

As will be illustrated below, the $S_{1}, S_{2}$, and $S_{3}$ states can be approximated in terms of a simple four-electron-in-four-orbital model. The symetrical HOMO-1, HOMO, LUMO, and LUMO+1 of $\mathrm{m} 22$ will be denoted $b_{1}, a_{2}$, and $a_{2}^{*}$, and $b_{1}^{*}$, respectively. They read

$$
\begin{aligned}
b_{1} & =\frac{\ell+r}{\sqrt{2}}, \\
a_{2} & =\frac{\ell-r}{\sqrt{2}}, \\
a_{2}^{*} & =\frac{\ell^{*}-r^{*}}{\sqrt{2}}, \\
b_{1}^{*} & =\frac{\ell^{*}+r^{*}}{\sqrt{2}},
\end{aligned}
$$

where $\ell$ and $\ell^{*}$ denote the pseudofragment orbitals localised on the left and $r$ and $r^{*}$ the same on the right (see Figure 7). As already mentioned, they are almost identical to the frontier orbitals of two isolated p2 species (see Figure 8). Note that the orthogonalisation tails of $\ell, \ell^{*}, r$, and $r^{*}$, constructed as orthogonal orbitals via a $45^{\circ}$-rotation of $b_{1}, a_{2}, a_{2}^{*}$, and $b_{1}^{*}$ are tiny (they are not even visible in Figure 7), which means that fully localised, nonorthogonal orbitals would have negligible overlaps (in other words, $\ell, \ell^{*}, r$, and $r^{*}$ are almost fully localised).

Let us then introduce the singlet configuration-state functions (CSFs) made of single excitations within the four-orbital subset. The four delocalised CSFs read

$$
\begin{aligned}
\Phi_{b a} & =\frac{\left|b_{1} \overline{a_{2}^{*}}\right|+\left|a_{2}^{*} \overline{b_{1}}\right|}{\sqrt{2}}, \\
\Phi_{a b} & =\frac{\left|a_{2} \overline{b_{1}^{*}}\right|+\left|b_{1}^{*} \overline{a_{2}}\right|}{\sqrt{2}}, \\
\Phi_{a a} & =\frac{\left|a_{2} \overline{a_{2}^{*}}\right|+\left|a_{2}^{*} \overline{a_{2}}\right|}{\sqrt{2}}, \\
\Phi_{b b} & =\frac{\left|b_{1} \overline{b_{1}^{*}}\right|+\left|b_{1}^{*} \overline{b_{1}}\right|}{\sqrt{2}} .
\end{aligned}
$$

They correspond to $\mathrm{HOMO}-1\left(b_{1}\right) \rightarrow \operatorname{LUMO}\left(a_{2}^{*}\right), \operatorname{HOMO}\left(a_{2}\right) \rightarrow \mathrm{LUMO}+$ $1\left(b_{1}^{*}\right), \mathrm{HOMO}\left(a_{2}\right) \rightarrow \operatorname{LUMO}\left(a_{2}^{*}\right)$, and $\mathrm{HOMO}-1\left(b_{1}\right) \rightarrow \mathrm{LUMO}+1\left(b_{1}^{*}\right)$, respectively. Lower-lying doubly occupied orbitals are implicit in the Slater determinants involved in Eq. 2. 
The corresponding localised CSFs read

$$
\begin{aligned}
\Phi_{\ell \ell} & =\frac{\left|\ell \overline{\ell^{*}}\right|+\left|\ell^{*} \bar{\ell}\right|}{\sqrt{2}}, \\
\Phi_{r r} & =\frac{\left|r \overline{r^{*}}\right|+\left|r^{*} \bar{r}\right|}{\sqrt{2}}, \\
\Phi_{\ell r} & =\frac{\left|\ell \overline{r^{*}}\right|+\left|r^{*} \bar{\ell}\right|}{\sqrt{2}}, \\
\Phi_{r \ell} & =\frac{\left|r \overline{\ell^{*}}\right|+\left|\ell^{*} \bar{r}\right|}{\sqrt{2}} .
\end{aligned}
$$

The two sets are related through

$$
\begin{aligned}
\Phi_{b a} & =\frac{\Phi_{\ell \ell}-\Phi_{r r}-\Phi_{\ell r}+\Phi_{r \ell}}{2}, \\
\Phi_{a b} & =\frac{\Phi_{\ell \ell}-\Phi_{r r}+\Phi_{\ell r}-\Phi_{r \ell}}{2}, \\
\Phi_{a a} & =\frac{\Phi_{\ell \ell}+\Phi_{r r}-\Phi_{\ell r}-\Phi_{r \ell}}{2}, \\
\Phi_{b b} & =\frac{\Phi_{\ell \ell}+\Phi_{r r}+\Phi_{\ell r}+\Phi_{r \ell}}{2} .
\end{aligned}
$$

The localised CSFs obviously play the role of zero-order, localised diabatic states of pure LE or CT type,

$$
\begin{aligned}
\Psi_{\mathrm{LE} \ell \ell}^{\text {dia0 }} & =\Phi_{\ell \ell}, \\
\Psi_{\mathrm{LE} r r}^{\text {dia }} & =\Phi_{r r}, \\
\Psi_{\mathrm{CT} \ell r}^{\text {dia }} & =\Phi_{\ell r}, \\
\Psi_{\mathrm{CT} r \ell}^{\mathrm{dia} 0} & =\Phi_{r \ell} .
\end{aligned}
$$

Delocalised diabatic states of pure LE or CT type are then obtained from the latter via a $45^{\circ}$-rotation,

$$
\begin{gathered}
\Psi_{\mathrm{LE}-}^{\mathrm{dia} 0}=\frac{\Psi_{\mathrm{LE} \ell \ell}^{\mathrm{dia} 0}-\Psi_{\mathrm{LE} r r}^{\mathrm{dia} 0}}{\sqrt{2}}=\frac{\Phi_{\ell \ell}-\Phi_{r r}}{\sqrt{2}}=\frac{\Phi_{b a}+\Phi_{a b}}{\sqrt{2}}, \\
\Psi_{\mathrm{LE}+}^{\mathrm{dia} 0}=\frac{\Psi_{\mathrm{LE} \ell \ell}^{\mathrm{dia} 0}+\Psi_{\mathrm{LE} r r}^{\mathrm{dia} 0}}{\sqrt{2}}=\frac{\Phi_{\ell \ell}+\Phi_{r r}}{\sqrt{2}}=\frac{\Phi_{a a}+\Phi_{b b}}{\sqrt{2}}, \\
\Psi_{\mathrm{CT}-}^{\mathrm{dia} 0}=\frac{\Psi_{\mathrm{C} \ell \ell}^{\mathrm{dia} \ell}-\Psi_{\mathrm{CT} r \ell}^{\mathrm{dia} 0}}{\sqrt{2}}=\frac{\Phi_{\ell r}-\Phi_{r \ell}}{\sqrt{2}}=\frac{-\Phi_{b a}+\Phi_{a b}}{\sqrt{2}}, \\
\Psi_{\mathrm{CT}+}^{\mathrm{dia} 0}=\frac{\Psi_{\mathrm{CT} \ell r}^{\mathrm{dia} \ell}+\Psi_{\mathrm{C} T r \ell}^{\mathrm{dia} 0}}{\sqrt{2}}=\frac{\Phi_{\ell r}+\Phi_{r \ell}}{\sqrt{2}}=\frac{-\Phi_{a a}+\Phi_{b b}}{\sqrt{2}} .
\end{gathered}
$$

Note that each delocalised CSF is thus an equal mixture of both LE and CT contributions. 
At the $S_{0}\left(1^{1} A_{1}\right)$ minimum, $\min _{S_{0}}$, the first three adiabatic excited states, $S_{1}\left(1^{1} B_{2}\right.$, bright: $\left.f=1.707\right), S_{2}\left(2^{1} A_{1}\right.$, bright: $\left.f=0.367\right)$, and $S_{3}\left(1^{1} A_{1}\right.$, dark: $f=0.001$ ), result mainly from combinations of two single excitations involving the four near-frontier orbitals discussed above, as shown in Table 2. The fourth related state (within the space generated by the four CSFs) is $S_{8}\left(4^{1} A_{1}\right.$, almost dark: $f=0.018)$ at $5.45 \mathrm{eV}(f$ denote the oscillator strength $)$.

\begin{tabular}{c|cc|cc|}
\multirow{5}{*}{} & \multicolumn{2}{|c|}{$\min _{S_{0}}$} & \multicolumn{2}{c|}{$\min _{S_{1}}$} \\
\cline { 2 - 5 }$S_{1}$ & deloc. CSF & coefficient & loc. CSF \\
\hline & $\Phi_{b a}$ & 0.71 & $\sim \Phi_{\ell \ell}$ & -0.03 \\
& $\Phi_{a b}$ & 0.66 & $\sim \Phi_{r r}$ & 0.91 \\
& & & $\sim \Phi_{\ell r}$ & 0.26 \\
& & & $\sim \Phi_{r \ell}$ & 0.23 \\
& & $(94 \%)$ & & $(94 \%)$ \\
\hline \multirow{5}{*}{$S_{2}$} & $\Phi_{a a}$ & 0.87 & $\sim \Phi_{\ell \ell}$ & 0.08 \\
& $\Phi_{b b}$ & 0.41 & $\sim \Phi_{r r}$ & 0.05 \\
& & & $\sim \Phi_{\ell r}$ & -0.65 \\
& & & $\sim \Phi_{r \ell}$ & 0.53 \\
& & $(92 \%)$ & & $(72 \%)$ \\
\hline \multirow{5}{*}{$S_{3}$} & $\Phi_{b a}$ & -0.55 & $\sim \Phi_{\ell \ell}$ & 0.60 \\
& $\Phi_{a b}$ & 0.60 & $\sim \Phi_{r r}$ & 0.27 \\
& & & $\sim \Phi_{\ell r}$ & -0.42 \\
& & & $\sim \Phi_{r \ell}$ & -0.56 \\
& & & & $(93 \%)$ \\
\hline \multirow{5}{*}{$S_{n}$} & $\Phi_{a a}$ & -0.41 & $\sim \Phi_{\ell \ell}$ & 0.72 \\
& $\Phi_{b b}$ & 0.85 & $\sim \Phi_{r r}$ & -0.20 \\
& & & $\sim \Phi_{\ell r}$ & 0.41 \\
& & & $\sim \Phi_{r \ell}$ & 0.38 \\
& & $(89 \%)$ & & $(86 \%)$ \\
\hline
\end{tabular}

Table 2: Coefficients of the singlet CSFs, restricted to the four near-frontier orbitals, contributing to the first three excited states of $\mathrm{m} 22$ at $\min _{S_{0}}$ and $\min _{S_{1}}$ and to the fourth related state $S_{n}\left(n=8\right.$ at $\min _{S_{0}}$ and $n=7$ at $\left.\min _{S_{1}}\right)$. The global weight of such contributions within the full configuration-interaction-like TD-DFT expansion is indicated within parentheses.Note that $S_{4}$ to $S_{7}$ at $\min _{S_{0}}$ are dark valence states involving noninteracting $\pi$ orbitals on both external benzene rings and in-plane $\pi / \pi^{*}$ acetylene orbitals. They correlate at $\min _{S_{1}}$ with $S_{4}$ to $S_{6}$, and $S_{8}$, where excitations are of LE character within p2 pseudofragments.

The TD-DFT configuration-interaction-like coefficients displayed in Table 2 show that each of the four states is an almost balanced combination of two 
delocalised CSFs. They can be recast as

$$
\begin{gathered}
\Psi_{1}^{\text {adia }}\left(\min _{S_{0}}\right)=0.97\left(\cos 43^{\circ} \Phi_{b a}+\sin 43^{\circ} \Phi_{a b}\right)+\ldots, \\
\Psi_{2}^{\text {adia }}\left(\min _{S_{0}}\right)=0.96\left(\cos 25^{\circ} \Phi_{a a}+\sin 25^{\circ} \Phi_{b b}\right)+\ldots, \\
\Psi_{3}^{\text {adia }}\left(\min _{S_{0}}\right)=0.81\left(-\sin 42^{\circ} \Phi_{b a}+\cos 42^{\circ} \Phi_{a b}\right)+\ldots, \\
\Psi_{8}^{\text {adia }}\left(\min _{S_{0}}\right)=0.94\left(-\sin 26^{\circ} \Phi_{a a}+\cos 26^{\circ} \Phi_{b b}\right)+\ldots
\end{gathered}
$$

For each state, the deviation of the global rescaling factor (e.g., 0.97 for $S_{1}$; its square, $94 \%$, is the global weigth given in Table 2) with respect to an ideal value of 1 is a measure of the contributions of other CSFs than the four dominant ones spanning the four-state model subspace. Such contributions are small (slightly less so for the $S_{3}$ state). After rescaling (partial renormalisation), it is clear from Eq. 7 that $S_{1}$ and $S_{3}\left(B_{2}\right.$ states $)$ are rotated together within the $\left\{\Phi_{b a}, \Phi_{a b}\right\}$ subspace through about $42-43^{\circ}$, while $S_{2}$ and $S_{8}$ ( $A_{1}$ states) are rotated together within the $\left\{\Phi_{a a}, \Phi_{b b}\right\}$ subspace through about $25-26^{\circ}$. Angle values of $45^{\circ}$ would correspond to the delocalised diabatic states of pure LE or CT type defined in Eq. 6. In other words, the deviation from $45^{\circ}$ is a measure of the admixture between LE and CT contributions. The adiabatic states can thus be recast in terms of the zero-order diabatic states,

$$
\begin{gathered}
\Psi_{1}^{\text {adia }}\left(\min _{S_{0}}\right)=0.97\left(\cos 2^{\circ} \Psi_{\mathrm{LE}-}^{\text {dia0 }}-\sin 2^{\circ} \Psi_{\mathrm{CT}-}^{\text {dia } 0}\right)+\ldots, \\
\Psi_{2}^{\text {adia }}\left(\min _{S_{0}}\right)=0.96\left(\cos 20^{\circ} \Psi_{\mathrm{LE}+}^{\text {dia0 }}-\sin 20^{\circ} \Psi_{\mathrm{CT}+}^{\text {dia }}\right)+\ldots, \\
\Psi_{3}^{\text {adia }}\left(\min _{S_{0}}\right)=0.81\left(\sin 3^{\circ} \Psi_{\mathrm{LE}-}^{\text {dia }}+\cos 3^{\circ} \Psi_{\mathrm{CT}-}^{\text {dia }}\right)+\ldots, \\
\Psi_{8}^{\text {adia }}\left(\min _{S_{0}}\right)=0.94\left(\sin 19^{\circ} \Psi_{\mathrm{LE}+}^{\text {dia }}+\cos 19^{\circ} \Psi_{\mathrm{CT}+}^{\text {dia }}\right)+\ldots,
\end{gathered}
$$

i.e.,

$$
\begin{aligned}
& \Psi_{1}^{\text {adia }}\left(\min _{S_{0}}\right)=0.97 \Psi_{\mathrm{LE}-}^{\text {dia }}-0.04 \Psi_{\mathrm{CT}-}^{\text {dia } 0}+\ldots \\
& \Psi_{2}^{\text {adia }}\left(\min _{S_{0}}\right)=0.90 \Psi_{\mathrm{LE}+}^{\text {dia }}-0.33 \Psi_{\mathrm{CT}+}^{\text {dia }}+\ldots \\
& \Psi_{3}^{\text {adia }}\left(\min _{S_{0}}\right)=0.04 \Psi_{\mathrm{LE}-}^{\text {dia0 }}+0.81 \Psi_{\mathrm{CT}-}^{\text {dia }}+\ldots \\
& \Psi_{8}^{\text {adia }}\left(\min _{S_{0}}\right)=0.31 \Psi_{\mathrm{LE}+}^{\text {dia }}+0.89 \Psi_{\mathrm{CT}+}^{\text {dia } 0}+\ldots
\end{aligned}
$$

Such relationships correspond de facto to originating a diabatisation "by hand" at the $\min _{S_{0}}$ point. The diabatic representation is based on zero-order diabatic states (model subspace) but could be turned into a more refined effectiveHamiltonian model (yielding the same eigenvalues as the target subspace defined by the four adiabatic states) upon relaxing the definition of the diabatic states.

Squaring the coefficients yields the LE or CT character of each state: $S_{1}$ is $93 \% \mathrm{LE}, 0.2 \% \mathrm{CT}$ (and $6 \%$ due to external CSF contributions); $S_{2}$ is $82 \% \mathrm{LE}$, $11 \% \mathrm{CT}$ (and $8 \%$ due to external CSF contributions); $S_{3}$ is $66 \% \mathrm{CT}, 0.2 \% \mathrm{LE}$ (and $34 \%$ due to external CSF contributions); $S_{8}$ is $80 \% \mathrm{CT}, 10 \% \mathrm{LE}$ (and $11 \%$ due to external CSF contributions). Note that the sum of such contributions do not seem to sum up to $100 \%$ only because of rounding. This situation holds all over the $\mathcal{C}_{2 \mathrm{v}}$ subspace, in particular at the $\mathrm{CoIn}_{S_{2} / S_{1}}, \mathrm{TS}_{B_{2}}$, and $\mathrm{TS}_{A_{1}}$ points, 
except that $S_{1}$ and $S_{2}$ swap their diabatic characters from one to the other side of the conical intersection.

Let us now analyse the composition of the adiabatic states at one of the two equivalent $S_{1}$ minima, $\min _{S_{1}}$. The HOMO and LUMO orbitals are rather localised on the excited p2 pseudofragment (cumulenic bonding pattern and corresponding geometry), while $\mathrm{HOMO}-1$ and $\mathrm{LUMO}+1$ are rather localised on the unexcited p2 pseudofragment (alternated) (see Figure 10). Note that HOMO and LUMO are more localised than the other two. The $S_{1}$ state (bright: $f=$ $1.133)$ corresponds to an almost pure ( $82 \%)$ HOMO $\rightarrow$ LUMO single excitation, which clearly is an LE state on the excited p2 pseudofragment. As already mentioned, the $S_{2}$ and $S_{3}$ states have crossed at a weakly avoided crossing along the path from the conical intersection to the $S_{1}$ minimum and thus swapped their diabatic characters. The $S_{3}$ state (bright: $f=0.931$ ) is dominated by the other LE state (HOMO-1 $\rightarrow$ LUMO $+1,36 \%$ ) on the p2 pseudofragment having a ground-state geometry, with significant CT contributions, while the $S_{2}$ state (dark: $f=0.015$ ) is almost a pure combination of the two CT states, correlating with the dark $S_{3}\left(1^{1} A_{1}\right)$ at the FC point. The fourth related state (within the space generated by the four CSFs) is $S_{7}\left(7^{1} A^{\prime}\right.$, almost dark: $\left.f=0.034\right)$ at 5.46 $\mathrm{eV}$. It also is dominated by the other LE state (HOMO-1 $\rightarrow \mathrm{LUMO}+1,51 \%$ ).

At the $S_{1}$ minimum, the four near-frontier orbitals are only approximately localised (see Figure 10), so that the interpretation is not so clear as for the $S_{0}$ minimum where symmetry helps. However, analysing other properties supports the diabatic picture discussed above. In particular, a comparison between the energies of $\mathrm{m} 22$ and $\mathrm{p} 2$ reflect that $\mathrm{m} 22$ behaves as a pair of weakly coupled $\mathrm{p} 2$ chromophores, and not only at high-symmetry points. The ground-state energy at the lower-symmetry $S_{1}$ minimum (see Table 1 ) is $0.32 \mathrm{eV}$ for both m22 and $\mathrm{p} 2$. In other words, varying the geometry of $\mathrm{m} 22$ from the $S_{0}$ minimum to the $S_{1}$ minimum requires the same energy as for a single p2 subunit, in agreement with the pseudofragmentation scheme. At the $S_{1}$ minimum of m22, the vertical transition energy of the first LE state $S_{1}$ (excitation of the p2 subunit that is in its excited-state geometry) is $3.80 \mathrm{eV}$, while the vertical transition energy of a bare p2 at its $S_{1}$ minimum is $3.82 \mathrm{eV}$. The vertical transition of the second LE state $S_{3}$ (excitation of the other p2 subunit, being in its ground-state geometry) is $4.42 \mathrm{eV}$, while the vertical transition energy of a bare p2 at its $S_{0}$ minimum is $4.48 \mathrm{eV}$.

This property is not coincidental. For example, we calculated the energies of $S_{1}$ and $S_{2}$ from the FC point when varying the length of one of the two acetylenic bonds in m22 and compared it to the $S_{0}$ and $S_{1}$ energies of a bare p2 undergoing the same geometric change. The $S_{1}$ and $S_{2}$ vertical energies of $\mathrm{m} 22$ are 4.43 and $4.47 \mathrm{eV}$, respectively. The underlying diabatic states should thus cross at $4.45 \mathrm{eV}$ (for a bond length equal to $1.210 \AA$ ), with an off-diagonal coupling term equal to half the difference: $0.02 \mathrm{eV}$ (much as a resonance integral between two sites). The $S_{1}$ energy of p2 was thus shifted from 4.48 to $4.45 \mathrm{eV}$ (to account for the small effect of CT contributions in m22 on the $S_{1}$ and $S_{2}$ energies), while the $S_{0}$ energy of p2 was shifted to the energy of the crossing point too. As can be observed in Figure 12, the agreement is almost perfect, 
such that the $S_{1}$ and $S_{2}$ curves in m22 can be regarded indeed as resulting from a weakly avoided crossing between two p2 pseudofragments, one excited and the other not.

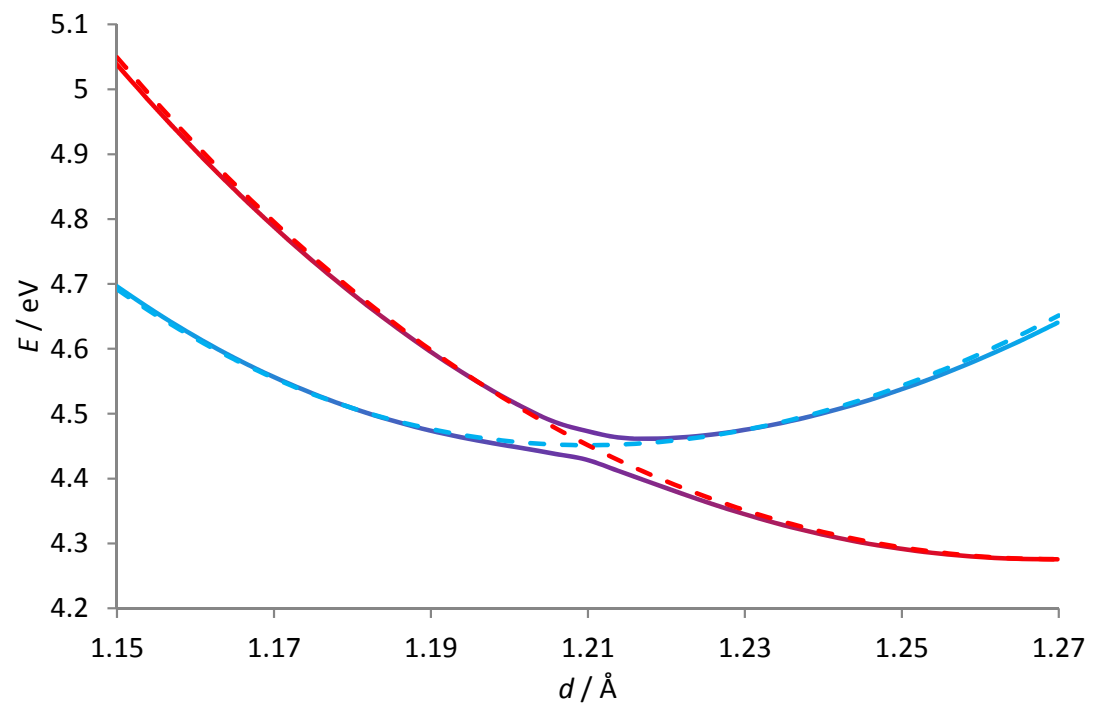

Figure 12: Evolution of the energies of the first two excited states of $\mathrm{m} 22$ along a variation of the bond length of one acetylenic bond, $d$, from its ground-state minimum (full lines). Evolution of the energies of the ground- and first-excited state of $\mathrm{p} 2$ along the same geometric change (dashed lines), shifted so as to make the curves coincide at the avoided crossing point: $d=1.21 \AA$ and $E=4.45 \mathrm{eV}$. 


\section{Conclusions}

We showed that the $S_{1}$ and $S_{2}$ states of m22 (meta-diphenylethynylphenylene) are essentially a pair of LE states that cross at a conical intersection. They can be described in terms of a two-state diabatic model (ignoring the CT states) based on a pseudofragmentation scheme involving two p2 (diphenylethynylene, also known as diphenylacetylene or tolane) subunits sharing a meta-substituted phenylene ring, owing to the fact that this site contributes with two pairs (two HOMOs and two LUMOs) of degenerate benzene orbitals.

Two equivalent zero-order diabatic representations can be used: the delocalised one is symmetry-adapted and coincides with the adiabatic one over the high-symmetry $C_{2 \mathrm{v}}$ subspace; the localised one coincides with the adiabatic one around both $S_{1}$ minima so as to rationalise the double-well topography of the $S_{1}$ PES as a symmetrical avoided crossing. The latter representation accounts for the small energy gap between $S_{1}$ and $S_{2}$ over the high-symmetry $C_{2 \mathrm{v}}$ subspace as resulting from a weak interaction between two identical p2 pseudofragments.

Localising the excitation on either one or the other pseudofragment is achieved upon breaking the symmetry to $C_{\mathrm{s}}$ so as to relax the geometry of the excited pseudofragment from a single-triple-single-bond (alternated) to a doubledouble-double-bond (cumulenic) geometry, according to the bonding pattern induced by transitions among frontier orbitals. Changing from the delocalised, $\left\{\Psi_{\mathrm{LE}-}^{\text {dia0 }}, \Psi_{\mathrm{LE}+}^{\mathrm{dia} 0}\right\}$, for the localised, $\left\{\Psi_{\mathrm{LE} \ell \ell}^{\mathrm{dia} 0}, \Psi_{\mathrm{LE} r r}^{\mathrm{dia} 0}\right\}$, diabatic representation, using Eq. 6, corresponds to a mere $45^{\circ}$-rotation (normalised sum and difference), known in this context as a Nikitin transformation [28, 29, 30].

The m22 species is the smallest subunit of PPE dendrimers for which EET can be envisioned (single and unique meta-substitution between a pair of the simplest linear peudofragments, p2). Due to symmetry, there will be no net EET nor net CT in this system from a dynamical point of view, as such processes selfcompensate symmetrically between the left and right sides (the two localised minima are mirror images of each other). However, this molecule is of interest on its own sake, as it plays the role of the "leaves" of the "nano-trees" that PPE dendrimers are expected to mimick. Also, m22 is a prototype for lessersymmetrical situations. Work is in progress, with a similar study being carried out on m23 (to be submitted soon), whereby we identified a weakly avoided crossing between $S_{1}$ and $S_{2}$, now related to a nonsymmetrical double well, in perfect agreement with unidirectional EET regarded as nonadiabatic internal conversion between LE states attached to either p2 or p3 pseudofragments (see Figure 13). Our pseudofragmentation procedure is thus expected to be general for the definition of practical diabatic representations in this context, and a prerequisite for further nonadiabatic quantum-dynamics simulations of EET in multichromophoric assemblies.

As a final word, let us mention that we focussed our study on in-plane bond-stretching distortions, as such motions most directly affect the electronic structure due to correlated changes in the bonding patterns. However, the effect

of soft modes such as local torsions and global (phonon-like) warpings deserve special attention, as they will modulate $\pi$-conjugation. Along a similar line, let 

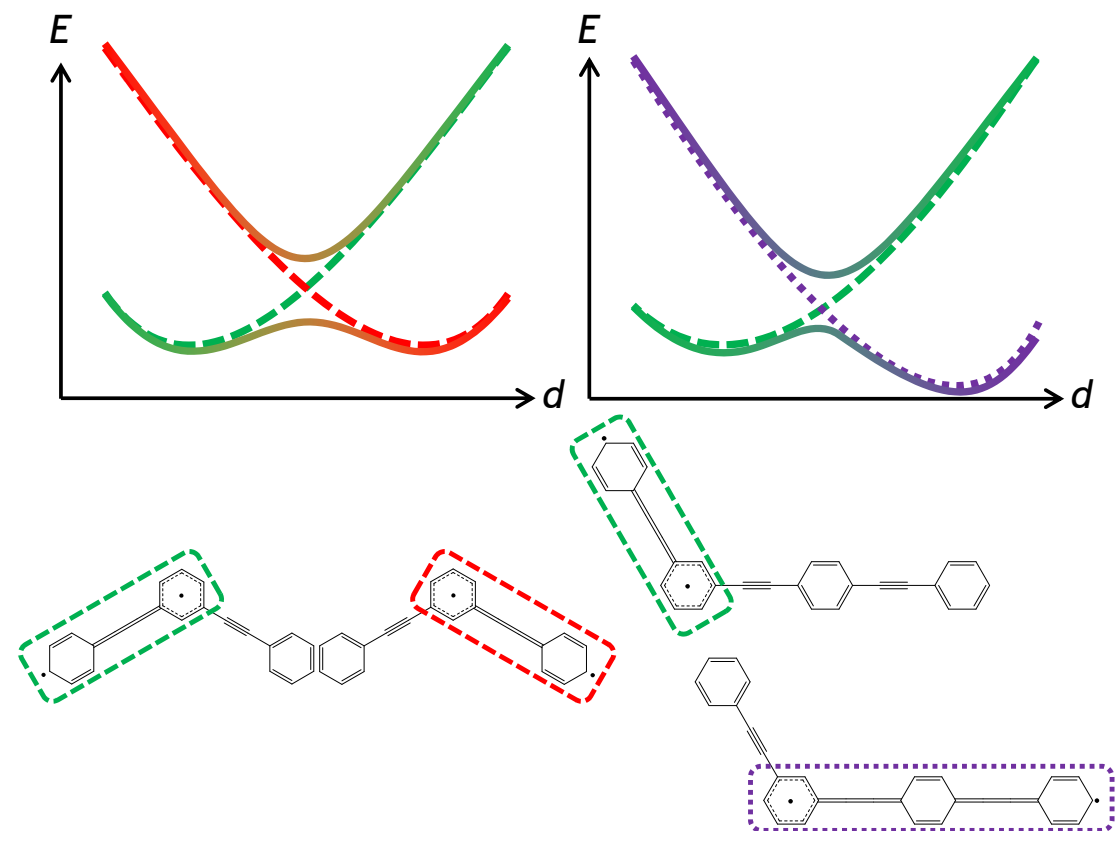

Figure 13: Schematic representation of symmetrical (m22, left) and nonsymmetrical (m23, right) weakly avoided crossings responsible of EET in metasubstituted PPE subunits. 
us mention that in-plane "zigzag" bending modes of CCCC chains may induce significant changes in the nature of the electronic states, as hinted for the $\mathrm{p} 2$ species in Ref. [31]. Such open questions will be addressed in future work, involving nonadiabatic quantum dynamics.

\section{Supplementary Information}

The Cartesian coordinates of the two p2 and five $\mathrm{m} 2$ critical points discussed above are provided in Supplementary Information.

\section{Acknowledgments}

Both authors warmly thank Marie-Liesse Doublet for her continued interest in this work, providing useful advice and fruitful discussions. EKLH is grateful to the French MENRT for funding her PhD grant.

\section{References}

[1] H. Köppel, W. Domcke, and L. S. Cederbaum. Multimode molecular dynamics beyond the Born-Oppenheimer approximation. Advances in Chemical Physics, 57:59-246, 1984.

[2] G. A. Worth, H.-D. Meyer, H. Köppel, and L. S. Cederbaum. Using the MCTDH wavepacket propagation method to describe multimode nonadiabatic dynamics. International Reviews in Physical Chemistry, 27:569606, 2008.

[3] A. Raab, G. Worth, H.-D. Meyer, and L. S. Cederbaum. Molecular dynamics of pyrazine after excitation to the $\mathrm{S}_{2}$ electronic state using a realistic 24-mode model Hamiltonian. The Journal of Chemical Physics, 110:936946, 1999.

[4] Aurelie Perveaux, Pedro J. Castro, David Lauvergnat, Mar Reguero, and Benjamin Lasorne. Intramolecular charge transfer in 4-aminobenzonitrile does not need the twist and may not need the bend. The Journal of Physical Chemistry Letters, 6(8):1316-1320, 2015. PMID: 26263129.

[5] V. May and O. Kühn. Charge and Energy Transfer Dynamics in Molecular Systems. WILEY-VCH, Weinheim, 2004.

[6] Zhi-Qiang You and Chao-Ping Hsu. Theory and calculation for the electronic coupling in excitation energy transfer. International Journal of Quantum Chemistry, 114(2):102-115, 2014.

[7] Cloé Azarias, Lorenzo Cupellini, Anouar Belhboub, Benedetta Mennucci, and Denis Jacquemin. Modelling excitation energy transfer in covalently 
linked molecular dyads containing a bodipy unit and a macrocycle. Phys. Chem. Chem. Phys., 20:1993-2008, 2018.

[8] Zhifu Xu and J. S. Moore. Design and synthesis of a convergent and directional molecular antenna. Acta Polymerica, 45(2):83-87, 1994.

[9] Takahito Oyamada, Guang Shao, Hiroyuki Uchiuzou, Hajime Nakanotani, Akihiro Orita, Junzo Otera, Masayuki Yahiro, and Chihaya Adachi. Optical and Electrical Properties of Bis(4-(phenylethynyl)phenyl)ethynes and Their Application to Organic Field-Effect Transistors. Japanese Journal of Applied Physics, 45(No. 50):L1331-L1333, December 2006.

[10] Raymond Ziessel, Gilles Ulrich, Alexandre Haefele, and Anthony Harriman. An Artificial Light-Harvesting Array Constructed from Multiple Bodipy Dyes. Journal of the American Chemical Society, 135(30):11330-11344, July 2013.

[11] Chelladurai Devadoss, P. Bharathi, and Jeffrey S. Moore. Energy transfer in dendritic macromolecules: molecular size effects and the role of an energy gradient. Journal of the American Chemical Society, 118(40):9635-9644, 1996.

[12] Michael R. Shortreed, Stephen F. Swallen, Zhong-You Shi, Weihong Tan, Zhifu Xu, Chelladurai Devadoss, Jeffrey S. Moore, and Raoul Kopelman. Directed energy transfer funnels in dendrimeric antenna supermolecules. The Journal of Physical Chemistry B, 101(33):6318-6322, 1997.

[13] S. F. Swallen, R. Kopelman, J. S. Moore, and C. Devadoss. Dendrimer photoantenna supermolecules: energetic funnels, exciton hopping and correlated excimer formation. Journal of Molecular Structure, 485:585-597, 1999.

[14] Devens Gust. Very small arrays. Nature, 386:21-22, 1997.

[15] Raoul Kopelman, Michael Shortreed, Zhong-You Shi, Weihong Tan, Zhifu $\mathrm{Xu}$, Jeffrey S. Moore, Arie Bar-Haim, and Joseph Klafter. Spectroscopic evidence for excitonic localization in fractal antenna supermolecules. Physical Review Letters, 78(7):1239-1242, 1997.

[16] Valeria D. Kleiman, Joseph S. Melinger, and Dale McMorrow. Ultrafast Dynamics of Electronic Excitations in a Light-Harvesting Phenylacetylene Dendrimer. The Journal of Physical Chemistry B, 105(24):5595-5598, June 2001.

[17] Joseph S. Melinger, Yongchun Pan, Valeria D. Kleiman, Zhonghua Peng, Benjamin L. Davis, Dale McMorrow, and Meng Lu. Optical and Photophysical Properties of Light-Harvesting Phenylacetylene Monodendrons Based on Unsymmetrical Branching. Journal of the American Chemical Society, 124(40):12002-12012, October 2002. 
[18] Julio L. Palma, Evrim Atas, Lindsay Hardison, Todd B. Marder, Jonathan C. Collings, Andrew Beeby, Joseph S. Melinger, Jeffrey L. Krause, Valeria D. Kleiman, and Adrian E. Roitberg. Electronic Spectra of the Nanostar Dendrimer: Theory and Experiment . The Journal of Physical Chemistry C, 114(48):20702-20712, December 2010.

[19] S. Fernandez-Alberti, Adrian E. Roitberg, Valeria D. Kleiman, T. Nelson, and S. Tretiak. Shishiodoshi unidirectional energy transfer mechanism in phenylene ethynylene dendrimers. The Journal of Chemical Physics, 137(22):22A526, 2012.

[20] Johan F. Galindo, Evrim Atas, Aysun Altan, Daniel G. Kuroda, Sebastian Fernandez-Alberti, Sergei Tretiak, Adrian E. Roitberg, and Valeria D. Kleiman. Dynamics of Energy Transfer in a Conjugated Dendrimer Driven by Ultrafast Localization of Excitations. Journal of the American Chemical Society, 137(36):11637-11644, September 2015.

[21] Sebastian Fernandez-Alberti, Dmitry V. Makhov, Sergei Tretiak, and Dmitrii V. Shalashilin. Non-adiabatic excited state molecular dynamics of phenylene ethynylene dendrimer using a multiconfigurational Ehrenfest approach. Physical Chemistry Chemical Physics, 18(15):10028-10040, 2016.

[22] Tammie Nelson, Sebastian Fernandez-Alberti, Adrian E. Roitberg, and Sergei Tretiak. Electronic Delocalization, Vibrational Dynamics, and Energy Transfer in Organic Chromophores. J. Phys. Chem. Lett., 8(13):30203031, July 2017.

[23] Alexis L. Thompson, Kevin M. Gaab, Jianjun Xu, Christopher J. Bardeen, and Todd J. Martnez. Variable Electronic Coupling in Phenylacetylene Dendrimers: The Role of Frster, Dexter, and Charge-Transfer Interactions. The Journal of Physical Chemistry A, 108(4):671-682, January 2004.

[24] M. J. Frisch, G. W. Trucks, H. B. Schlegel, G. E. Scuseria, M. A. Robb, J. R. Cheeseman, G. Scalmani, V. Barone, G. A. Petersson, H. Nakatsuji, X. Li, M. Caricato, A. V. Marenich, J. Bloino, B. G. Janesko, R. Gomperts, B. Mennucci, H. P. Hratchian, J. V. Ortiz, A. F. Izmaylov, J. L. Sonnenberg, D. Williams-Young, F. Ding, F. Lipparini, F. Egidi, J. Goings, B. Peng, A. Petrone, T. Henderson, D. Ranasinghe, V. G. Zakrzewski, J. Gao, N. Rega, G. Zheng, W. Liang, M. Hada, M. Ehara, K. Toyota, R. Fukuda, J. Hasegawa, M. Ishida, T. Nakajima, Y. Honda, O. Kitao, H. Nakai, T. Vreven, K. Throssell, J. A. Montgomery, Jr., J. E. Peralta, F. Ogliaro, M. J. Bearpark, J. J. Heyd, E. N. Brothers, K. N. Kudin, V. N. Staroverov, T. A. Keith, R. Kobayashi, J. Normand, K. Raghavachari, A. P. Rendell, J. C. Burant, S. S. Iyengar, J. Tomasi, M. Cossi, J. M. Millam, M. Klene, C. Adamo, R. Cammi, J. W. Ochterski, R. L. Martin, K. Morokuma, O. Farkas, J. B. Foresman, and D. J. Fox. Gaussian16 Revision A.03, 2016. Gaussian Inc. Wallingford CT. 
[25] Emmeline Kim-Lien Ho, Thibaud Etienne, and Benjamin Lasorne. Vibronic properties of para-polyphenylene ethynylenes: TD-DFT insights. The Journal of Chemical Physics, 146(16):164303, April 2017.

[26] Jing Huang, Likai Du, Deping Hu, and Zhenggang Lan. Theoretical analysis of excited states and energy transfer mechanism in conjugated dendrimers. Journal of Computational Chemistry, 36(3):151-163, January 2015.

[27] Benjamin Gonon, Aurelie Perveaux, Fabien Gatti, David Lauvergnat, and Benjamin Lasorne. On the applicability of a wavefunction-free, energybased procedure for generating first-order non-adiabatic couplings around conical intersections. The Journal of Chemical Physics, 147(11):114114, 2017.

[28] E. E. Nikitin. Theory of Elementary Atomic and Molecular Processes in Gases. Clarendon, Oxford, 1974.

[29] M. Desouter-Lecomte, C. Galloy, J. C. Lorquet, and M. Vaz Pires. Nonadiabatic interactions in unimolecular decay. V. Conical and Jahn-Teller intersections. The Journal of Chemical Physics, 71:3661-3672, 1979.

[30] M. Desouter-Lecomte, D. Dehareng, B. Leyh-Nihant, M. T. Praet, A. J. Lorquet, and J. C. Lorquet. Nonadiabatic unimolecular reactions of polyatomic molecules. The Journal of Chemical Physics, 89:214-222, 1985.

[31] Christopher Robertson and Graham A. Worth. Modelling the non-radiative singlet excited state isomerization of diphenyl-acetylene: A vibronic coupling model. Chemical Physics, 510:17-29, 2018. 